\title{
Pensamiento computacional: una nueva exigencia para la educación del siglo XXI
}

\author{
Computational thinking: a new demand for education of the 21st century
}

\author{
Edith Soria Valencia* \\ Carol Rivero Panaqué
}

\section{Resumen}

En el actual siglo XXI, se requiere de ciudadanos preparados para enfrentar profundos cambios y retos, especialmente, en la vida y el trabajo. De esta manera, para una adecuada toma de decisiones en el mundo de hoy, complejo y cambiante, se necesitan desarrollar diferentes habilidades en las personas que les permitan desenvolverse adecuadamente. Por ello, la educación debe ser un pilar fundamental en la construcción de esta sociedad mediante la promoción de habilidades, como por ejemplo aquellas relacionadas al pensamiento computacional, considerando el pensamiento crítico y en donde se pueda descomponer un problema, procesar datos, crear procedimientos y generalizarlos. Por lo tanto, a través de este estudio, se reflexionará sobre la importancia del pensamiento computacional y se analizará sus aproximaciones conceptuales. Así también, se considerarán los elementos que lo componen y finalmente, se revisarán algunas experiencias de interés sobre la enseñanza del lenguaje de programación y pensamiento computacional.

Palabras clave: Pensamiento computacional. Educación. Resolución de problemas. Habilidades computacionales. Aprendizaje.

\section{Abstract}

In the current 21st century, citizens are required to face deep changes and challenges, especially in life and work. In this way, for an adequate decision making in today's world, the complex and the changing, skills must be developed for the people that allow them to develop properly. Thus, education must be a fundamental element in the construction of this society through the promotion of skills, for example, those related to computational thinking, critical thinking and the context in which a problem can be decomposed, data processing, create procedures and generalize them. Therefore, through this study, we will reflect on the importance of computational thinking and analyze it conceptually. Also, the elements that compose it will be considered and finally, some interesting experiences on the teaching of programming language and computational thinking were reviewed.

Keywords: Computational thinking. Education. Problem solving. Computer skills. Learning.

Recebido em 01/10/2018 - Aprovado em 24/01/2019

http://dx.doi.org/10.5335/rep.v26i2.8702

Doctora en Educación y Magíster en Educación. Coordinadora de Investigación de la Maestría en Integración e Innovación Educativa de las TIC na Pontifícia Universidad Católica del Perú, Perú. E-mail: edith.soria@pucp.pe

Doctoranda en Ciencias de la Educación. Magister en Ingeniería de Medios para la Educación por el Consorcio Euromime - Unión Europea. Profesora Asociada del Departamento de Educación de la Pontificia Universidad Católica del Perú. Directora de la Maestría en Integración e Innovación Educativa de las TIC - Pontifícia Universidad Católica del Perú, Perú. E-mail: crivero@pucp.pe 


\section{Introducción}

En la actual sociedad digital, los niños, jóvenes y adultos necesitan aprender y practicar nuevas habilidades, para lograr un mejor desenvolvimiento. Estos aprendizajes van relacionados al desarrollo de un pensamiento computacional orientados de forma creativa a generar nuevas posibilidades de respuesta ante los diversos problemas que surgen en un entorno que cambia constantemente debido a la incursión tecnológica.

De acuerdo con Balladares, Avilés y Pérez (2016), la tecnología está presente en diferentes contextos y en una amplia variedad de formas que se aproximan a los estudiantes en las diversas etapas del ciclo educativo. Entonces, pensar en un currículo adecuado a estas etapas educativas, lleva a reflexionar sobre los dominios conceptuales que demandará en cada una de ellas. Ello, también, permite considerar la capacitación de profesores y plantear un nuevo paradigma de enseñanza aprendizaje cuantitativo, intuitivo y de ensayo y error (RINCÓN; ÁVILA, 2016).

En este marco, se debería abordar de manera inteligente, los diversos problemas que se presentan en esta sociedad, utilizando técnicas y estructuras que se usan en las ciencias de la computación (BASOGAIN; OLABE; OLABE, 2015). Así, se partiría de la abstracción hacia el pragmatismo, transitando de las ideas hacia un mundo real. Por ello, el pensamiento computacional como nueva competencia del siglo XXI, debería ser incluida en la formación de todos las personas en las diferentes etapas educativas (educación básica y educación superior).

De todas maneras, pensar en la visibilización del pensamiento computacional en el sistema educativo demanda la inclusión de cambios normativos. De ahí que surgen ciertas limitaciones en su promoción, pues ello responde a decisiones políticas de las autoridades. Sin embargo, se hace necesario considerarlo, puesto que el pensamiento computacional ayuda a la toma decisiones de una manera ordenada, secuenciada, lógica, sin ambigüedades y permite la posibilidad de manipular objetos, transformarlos y crearlos, y convertir una idea en una acción, oportunidades potentes para facilitar la adquisición de habilidades.

\section{Referencia histórica sobre el pensamiento computacional}

Plantear el pensamiento computacional, como una estrategia de aprendizaje en pleno siglo XXI, nos remonta a la década de los ochenta (siglo XX), donde el desarrollo del aprendizaje de la geometría, fue la experiencia más próxima al tema, 
a partir de un lenguaje de programación denominado "Logo". En este esquema de los años 80, confluían las ideas de Dewey, Piaget y Vygotsky, las cuales fueron materializadas por Seymour Papert (VALVERDE; FERNÁNDEZ; GARRIDO, 2015) y generó una propuesta disruptiva en dicha década.

Revisando la literatura, "Logo" fue el primer lenguaje de programación basado en un enfoque pedagógico que permitió el aprendizaje de matemáticas, cibernética y ciencias de una forma innovadora. A partir de esta primera experiencia, surge años más tarde "Scratch", como un entorno de programación con gran potencialidad educativa (VALVERDE; FERNÁNDEZ; GARRIDO, 2015; PÉREZ; ROIG-VILA, 2015). En este punto es importante destacar que un objetivo clave del diseño de Scratch fue apoyar el aprendizaje autodidacta a través de la práctica personal y la colaboración con otros (BASOGAIN; OLABE; OLABE, 2015).

Este escenario inicial, tal como señala Zapata (2015), da cuenta de un proceso de alfabetización digital, donde se desarrolló un esfuerzo de adaptación a los nuevos medios de comunicación, representación y proceso de la información entre humanos. Sin embargo, es importante destacar que en la década de 1990, el término "alfabetización digital" tuvo un mayor tratamiento por diversos autores. Lo común fue plantear que la alfabetización digital significó esencialmente la capacidad de leer y comprender elementos de información en los formatos de hipertexto o multimedia. El desarrollo del hardware y el software durante este tiempo no brindó mayor variedad de aplicaciones y el usuario tenía que realizar diferentes tareas, lo cual complicaba su uso (ZAPATA, 2015). Por ello, no era un entorno favorable a la enseñanza y aprendizaje. Sin embargo, al paso del tiempo, el uso de los diversas aplicaciones y entornos se hizo más sencillo (VALVERDE; FERNÁNDEZ; GARRIDO, 2015) y este análisis evolutivo sobre la alfabetización digital, fue la antesala para el surgimiento de una nueva corriente, llamada "pensamiento computacional".

\section{Pensamiento computacional: una aproximación conceptual}

Las Tecnologías de la Información y la Comunicación, desde su aparición, han generado una serie de transformaciones vertiginosas en la sociedad. Las entidades educativas no son ajenas a este cambio, pues la dinámica pedagógica ha permitido generar un mayor protagonismo del alumno y el uso de estrategias metodológicas alternativas que desarrollan la creatividad de los estudiantes.

En dicho marco, diversos autores como Zapata (2015) y Rincón y Ávila (2016) han señalado la necesidad de promover una alfabetización digital orientada al 
aprendizaje de la programación y a la capacidad de utilizar las fuentes digitales de forma eficaz. Lo planteado, perfila la necesidad de incluir en la formación de los estudiantes, el desarrollo de una nueva competencia que debería ser lograda en la formación escolar de todos los estudiantes (ESPINO; GONZÁLEZ, 2015; ZAPATA, 2015). Ahora bien, la alfabetización digital no es exclusiva de los espacios formales, es evidente que también se aprende en ámbitos informales. Sin embargo, no basta solo con conocer o usar las tecnologías, sino que el estudiante debe incorporarlas a sus procesos de creación, innovación y gestión del conocimiento a través del pensamiento computacional (BALLADARES; AVILÉS; PÉREZ, 2016).

Una primera y errónea idea que se tiene del pensamiento computacional es suponer que es exclusivo de los ámbitos de la ingeniería informática y computación. En realidad, hay una relación directa con la educación pues existe mucho esfuerzo en incorporarlo en los proyectos, juegos, entornos de programación, etc., en el currículum de escuelas y universidades (BASOGAIN; OLABE; OLABE, 2015). Lamentablemente, la conceptualización siempre ha estado restringida al dominio de la tecnología o pulsaciones del teclado; y, en realidad, tiene que ver con el dominio de las ideas (ZAPATA, 2015). Por ello, la labor pedagógica para el desarrollo del pensamiento computacional, no puede ni debe estar orientado solo al desarrollo de habilidades técnicas; sino, al desarrollo de capacidades que permitan un pensamiento reflexivo y resolutivo de problemas que atienda las necesidades sociales de diferente tipo con ayuda de herramientas informáticas, que son tan comunes en nuestro medio (BALLADARES; AVILÉS; PÉREZ, 2016). En otras palabras, "el poder del pensamiento computacional no está en aprender a programar, está en entender cómo podemos expresar una idea utilizando una computadora o cualquier herramienta que permita insertar instrucciones" (RICO; BOSAGAIN, 2018, p. 30).

La International Society for Technology in Education (ISTE) y la Computer Science Teachers Association (CSTA), afirman que el pensamiento computacional es un enfoque para resolver un determinado problema que empodera la integración de las tecnologías digitales con ideas humanas (SÁEZ; CÓZAR, 2017; RINCÓN; ÁVILA, 2016; SEGREDO; MIRANDA; LEÓN, 2017) y tiene diversas características que corresponden al pensamiento científico (ESPINO; GONZÁLEZ, 2015). Por otro lado, Rojas y García (2018), afirman que el pensamiento computacional es un proceso cognitivo cuyo propósito es generar soluciones a los diversos problemas que se presentan, apoyados en el uso de la abstracción, la descomposición, el diseño algorítmico y, permite el desarrollo de habilidades como; el pensamiento crítico, la creatividad y la innovación (LLORENS et al., 2017). Esta forma de pensamiento 
se manifiesta en las múltiples actividades diarias que parten de tareas sencillas, como leer, realizar algún cálculo matemático y hasta dar solución a un problema (RICO; BASOGAIN, 2018; PALENCIA, 2017).

Como se aprecia, en los diversos aportes de los autores revisados sobre pensamiento computacional hay una constante que se mantiene presente en estas aproximaciones conceptuales, y se refiere a la necesidad de resolver problemas utilizando para ello diversos elementos como las matemáticas, el pensamiento crítico, la creatividad, la integración de las tecnologías digitales, la innovación y el trabajo colaborativo.

Partiendo de esta premisa, se puede diseñar un programa curricular basado en el pensamiento computacional; donde se enseñe a los niños, múltiples niveles de abstracción, se promuevan las habilidades no memorísticas o mecánicas, y se fortalezca la promoción de ideas creativas, que considere una interacción interdisciplinar. En otras palabras, se puede desarrollar en los estudiantes, el pensamiento crítico en alianza con los conceptos insertos en la computación, tales como abstracción, algoritmos, lenguajes de programación, simulación, modelos de estructura de datos e inteligencia artificial, entre otros (ZAPOTECATL, 2014).

\section{Habilidades del pensamiento computacional}

El pensamiento computacional es hoy en día un tema de mucha discusión (COMPAÑ et al., 2015), y cuyo mayor logro es potenciar habilidades relacionadas con la resolución creativa de problemas (PALENCIA, 2017). Estas habilidades, según Vilanova (2018) se apoyan en una serie de actitudes que son dimensiones esenciales del pensamiento computacional. Estas actitudes incluyen:

a) Confianza en uno mismo para el manejo de la complejidad;

b) Perseverancia al enfrentar problemas difíciles;

c) Tolerancia frente a situaciones ambiguas;

d) Habilidad para combatir problemas no estructurados.

Por lo tanto, las habilidades de un estudiante con pensamiento computacional supera la capacidad de la apropiación técnica y le incorpora habilidades para afrontar, entender y resolver problemas con mayor eficacia, lo cual le permita solucionar problemas en los diversos ámbitos de la vida (PALENCIA, 2017). De esta manera, es indispensable entender los conceptos técnicos respectivos de forma progresiva y contar con las actitudes necesarias para lograrlo. Estos dos elementos deben fortalecerse 
desde los primeros años de formación y, probablemente mucho antes que el niño(a) ingrese a la escuela, y luego, debe continuar en los diferentes niveles de la educación.

Vivimos una nueva etapa dondelas competencias relacionadas conla programación se consideran habilidades básicas; así como, la lecto-escritura y las matemáticas. Ello exige que el sistema educativo realice ajustes inmediatos ante este nuevo panorama (ESPINO; GONZÁLES, 2015). Bajo esta premisa, las entidades educativas tienen el gran reto de incluir un aprendizaje adicional; como es el desarrollo del pensamiento computacional, que les permita a los alumnos poder definir con facilidad criterios que los conduzcan de forma pertinente a la toma de decisiones (RINCÓN; ÁVILA, 2016). Es por tanto, una competencia compleja de alto nivel (VALVERDE; FERNÁNDEZ; GARRIDO, 2015), la cual se debería evidenciar en la actitud y las habilidades que todas las personas deberían tener y que no solo tendría que ser exclusividad de los profesionales informáticos (WING, 2008). Es claro, que la programación no es solo una competencia cognitiva, sino también, una competencia social y cultural que se usa para participar en grupos (VILANOVA, 2018).

De esta manera, el pensamiento computacional desarrolla diversas habilidades y procesos, como los que podemos ver a continuación desde los aportes de algunos autores:

Tabla 1 - Pensamiento computacional y fortalecimiento de habilidades

\begin{tabular}{|c|c|c|}
\hline Balladares, Avilés y Pérez (2016) & Rincón y Ávila (2016) & Sáez y Cózar (2017) \\
\hline $\begin{array}{l}\text { Analizar los efectos de la computación y definir } \\
\text { los alcances, ventajas así como limitaciones } \\
\text { que pueden presentar el uso de herramientas } \\
\text { informáticas para la solución de un problema. } \\
\text { Producir artefactos computacionales que per- } \\
\text { mitan producir las propias tecnologías. } \\
\text { Usar abstracción y modelos y a partir de ellos } \\
\text { construir modelos que permitan analizar o mo- } \\
\text { dificar sus condiciones. } \\
\text { Analizar problemas y artefactos, que permitan } \\
\text { resolver problemas. } \\
\text { Reconocer y generalizar patrones para clasi- } \\
\text { ficados. } \\
\text { Algoritmización, es decir organizar procesos } \\
\text { secuenciales lógicos de forma que resuelvan } \\
\text { problemas. } \\
\text { Comunicar procesos y resultados, que permi- } \\
\text { tan que la información sea puesta al servicio } \\
\text { de la sociedad. } \\
\text { Trabajar de forma efectiva en equipo compar- } \\
\text { tiendo experiencias e ideas. }\end{array}$ & $\begin{array}{l}\text { Formular problemas de tal ma- } \\
\text { nera que sea posible utilizar } \\
\text { computadoras y otras herra- } \\
\text { mientas para solucionarlos. } \\
\text { Organizar datos de manera ló- } \\
\text { gica y analizarlos. } \\
\text { Representar datos con el re- } \\
\text { curso de las abstracciones, } \\
\text { como modelos y simulaciones. } \\
\text { Automatizar soluciones me- } \\
\text { diante el pensamiento algorít- } \\
\text { mico. } \\
\text { Identificar, analizar e imple- } \\
\text { mentar posibles soluciones } \\
\text { para encontrar la combinación } \\
\text { más eficiente y efectiva de pa- } \\
\text { sos y recursos. } \\
\text { Generalizar y transferir el pro- } \\
\text { ceso de solución de problemas } \\
\text { a una gran diversidad de es- } \\
\text { tos. }\end{array}$ & $\begin{array}{l}\text { Pensar recursivamente. } \\
\text { Procesar en paralelo. } \\
\text { Generalizar el análisis di- } \\
\text { mensional. } \\
\text { Juzgar un programa por } \\
\text { simplicidad de diseño. } \\
\text { Utilizar abstracción y des- } \\
\text { composición en un pro- } \\
\text { blema complejo o diseño } \\
\text { de sistemas complejos. } \\
\text { Elegir una correcta repre- } \\
\text { sentación o modelo para } \\
\text { hacer tratable el proble- } \\
\text { ma. } \\
\text { Utilizar el razonamiento } \\
\text { heurístico para encontrar } \\
\text { la solución. }\end{array}$ \\
\hline
\end{tabular}

Nota: elaboración propia. Tomado de Balladares, Avilés y Pérez (2016); Rincón y Ávila (2016); Sáez y Cózar (2017). 
A la luz de estos aportes, se puede inferir que el desarrollo del pensamiento computacional permite promover múltiples habilidades y procesos, que no se reducen al dominio técnico de la programación; sino que, incorporan habilidades sociales. Además, el fortalecimiento del pensamiento computacional promueve un desarrollo cognitivo superior, donde la abstracción, iteración, aprendizaje colaborativo y meta cognición, terminan siendo sus componentes indispensables.

En relación al primer componente, la abstracción es la habilidad que se desarrolla a partir del pensamiento computacional, que permite crear y hacer uso de diferentes niveles de abstracción, para entender y resolver óptimamente los problemas (PÉREZ; ROIG-VILA, 2015). En tal sentido, "las abstracciones para la computación son 'las herramientas mentales' y las computadoras las herramientas 'metálicas' que automatizan estas abstracciones" (ZAPOTECATL, 2014, p. 9). Esto ocurre, pues las abstracciones son simbólicas y, por ello, tienden a ser más complejas que las de ciencias matemáticas y físicas (WING, 2008).

La iteración es un componente del pensamiento computacional y muchas veces, se asocia a procedimientos repetitivos. Sin embargo, es importante considerar que el diseño de un proyecto no es un proceso secuencial, es adaptativo. Por tanto, hay aprendizajes básicos, en las primeras etapas de desarrollo, donde se pone en marcha un sistema de pensamiento de este tipo (ZAPATA, 2015). El fortalecimiento del pensamiento computacional, se enriquece al paso de los años, llegando así a constituirse en un proceso cognitivo superior.

En cuanto al aprendizaje colaborativo, parece extraño pensar en la incursión de este término dentro del pensamiento computacional; sin embargo, la complejidad del desarrollo y arquitectura en el mundo computacional hace necesario pensar en la promoción de actividades colaborativas y distanciarse de la idea de un trabajo aislado. Generar nuevas ideas a partir del aprendizaje computacional, exige promover fuertes olas de trabajo y comunicación que permitan hacer posibles proyectos comunes. A partir de la premisa, se puede considerar que la formación en valores constituye un elemento indispensable en el desarrollo del pensamiento computacional (ZAPATA, 2015).

Otro proceso importante es la metacognición que permite a la persona plantear acciones para afrontar un problema y tomar decisiones para resolverlo. En el desarrollo del pensamiento computacional, este componente es clave, pues se considera como una estrategia que lo lleva a analizar y procesar toda la información relacionada en torno a un problema y con ello, a buscar soluciones. Por tanto, se requiere de una planificación de habilidades y destrezas que se aplican 
en función de las tareas a desarrollar. La metacognición exige que el estudiante tenga plena conciencia de la situación - problema sobre la cual va a aplicar algún nivel de respuesta. Asimismo, es necesario que el estudiante reconozca los recursos cognitivos con los que cuenta para hacer frente a dicha situación - problema.

\section{Pensamiento computacional en educación básica y educación superior ¿desarrollo cognitivo o perspectiva socioconstructivista en la educación?}

El aprendizaje es un proceso social y, la presencia de las tecnologías de la información y la comunicación perfilan una nueva ecología de aprendizaje, donde la motivación, la participación y la colaboración son indispensables pues la interacción de los estudiantes con la tecnología se genera en un contexto social (VALVERDE; FERNÁNDEZ; GARRIDO, 2015). Por ello, de acuerdo con Vega y Espinel (2010) es conveniente que el estudiante desde su formación inicial en la escuela conozca la importancia y significatividad de esta área del conocimiento como un medio para la resolución de problemas (PÉREZ; ROIG-VILA, 2015).

El pensamiento computacional no está dirigido solo al desarrollo de competencias cognitivas, como equivocadamente a veces se piensa; sino, que está orientado a generar una nueva ecología para el aprendizaje dentro de un contexto social. Por ello, el pensamiento computacional es una competencia social y cultural que invita al trabajo colaborativo.

Desde esta perspectiva, enseñar programación no consiste en enumerar una serie de estructuras de programación indicando para que sirve cada una de ellas. Se trata de que el estudiante aprenda a pensar, a analizar una situación y a diseñar el método de resolución más adecuado, dejando al margen el lenguaje de programación. Se trata de un objetivo muy complejo que requiere un esfuerzo importante de abstracción; aún más, si tiene que expresarla en forma de un algoritmo (COMPAÑ et al., 2015).

Por otra parte, Pérez y Roig-Vila (2015) señalan que promover el pensamiento computacional en estudiantes universitarios es una labor difícil debido a que la metodología de trabajo instruccional y los recursos tecnológicos educativos aplicados no permiten desarrollar apropiadamente muchas de sus habilidades como la abstracción y creatividad.

La programación en educación superior no se trata solo de escribir códigos, se busca analizar determinadas situaciones, identificar sus componentes, modelar 
los datos y procesos, y crear un programa. Es una tarea compleja que exige la participación de equipos de profesionales.

En educación, la programación se puede usar como herramienta de modelado para crear conocimiento y resolver problemas. Por ello, la programación debe considerarse como una estrategia pedagógica que orienta al estudiante a un proceso de co-creación de conocimiento creativo (ROMERO; LEPAGE; LILLE, 2017).

Como se muestra en la siguiente figura, existen cinco niveles de participación creativa en la programación: (1) exposición pasiva a explicaciones centradas en el profesor; (2) procedimientos paso a paso, con apoyo de tutoriales sobre programación; (3) creación de contenido original a través de la programación individual; (4) programación en equipo y finalmente; (5) co-creación participativa del conocimiento a través de la programación (ROMERO; LEPAGE; LILLE, 2017). Ver figura 1.

Figura 1 - Los cinco niveles de participación creativa en actividades de programación educativa

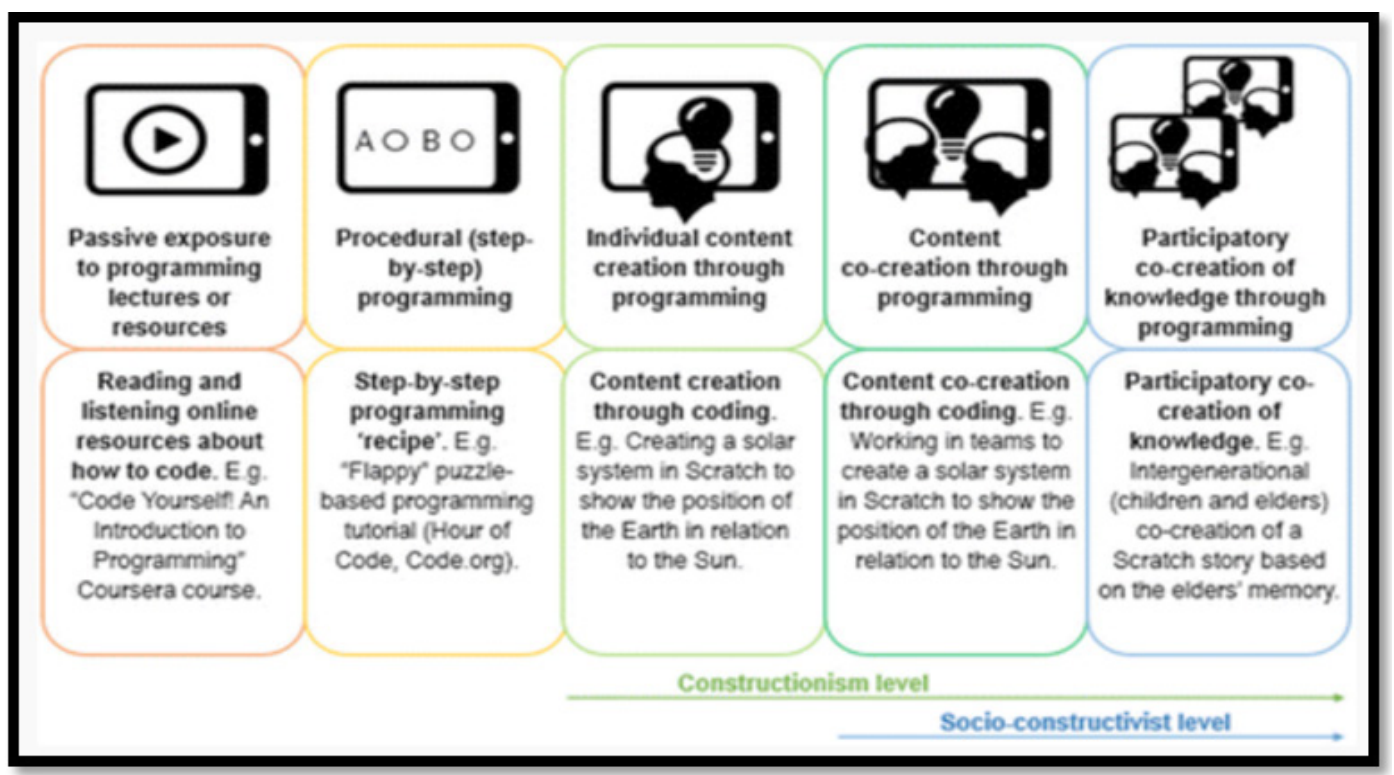

Fuente: tomado de Romero, Lepage y Lille (2017, p. 3).

Como podemos observar, la programación creativa requiere una participación activa del estudiante y sus pares, que los involucre en el proceso de diseño y desarrollo de co-construcción de conocimiento. 
Acontinuación, analizaremos algunas experiencias que pueden ser interesantes sobre la enseñanza de lenguaje de programación.

\section{Experiencias de interés sobre la enseñanza de lenguaje de programación}

Hoy en día se puede señalar que existen esfuerzos diversos por incorporar el pensamiento computacional en los sistemas educativos. Por ello, hay experiencias muy interesantes que se deben compartir para motivar nuevas iniciativas.

A nivel internacional, hay múltiples iniciativas que promueven la enseñanza de la programación en la escuela secundaria. Una experiencia interesante es la que brinda Estados Unidos, pues en el sistema educativo formal, ha integrado la asignatura de Programación a la cultura educativa. Por ello, para los estudiantes es habitual utilizar un lenguaje de programación como soporte a las Matemáticas, Física, Química o incluso áreas que no son exclusivas de Ciencias. Hay un gran esfuerzo en las escuelas por trabajar con herramientas que ayuden a una mejor didáctica de la Programación. De esta manera, se valora el uso de entornos gráficos que permitan el uso de estructuras lógicas y ayuden a desarrollar aplicaciones gráficas vistosas, motivando a los estudiantes con las exigencias de sus respectivas asignaturas y generando un progreso gradual en el aprendizaje (CARRALERO, 2011).

De la misma manera, ha surgido en este país, la iniciativa code.org ${ }^{1}$ que ha incorporado diversas herramientas didácticas cómo medio para enseñar programación. Esta iniciativa se desarrolló en Estados Unidos, y su propósito desde su creación ha estado orientado a promover la enseñanza de programación motivando a los estudiantes por el estudio de carreras a nivel superior relacionadas con el tema (BALLADARES; AVILÉS; PÉREZ, 2016).

En Estonia, la fundación "Tiger Leap Foundation" impulsa desde septiembre de 2012 un programa denominado "ProgeTiger", que estimula el aprendizaje de programación de computadoras y creación de aplicaciones web y móviles en la etapa de educación inicial. Esta iniciativa plantea diversos propósitos, como:

a) desarrollar entre los jóvenes el pensamiento computacional, la creatividad y las habilidades matemáticas; b) demostrar que la programación puede ser interesante y que cualquier persona puede aprender a programar; c) enseñar los fundamentos de la programación a través de la actividad práctica; y d) enseñar a los alumnos a utilizar diferentes lenguajes de programación adecuados a la edad (DÍAZ et al., 2014, p. 4). 
Otros países que se han sumado a estos programas son, Israel, Nueva Zelanda, Japón, Finlandia y Reino Unido, quienes tienen experiencias interesantes en torno al desarrollo del pensamiento computacional.

Inglaterra, por ejemplo, desde el 2014 ha incluido formalmente en el currículo educativo de la educación primaria y secundaria, el estudio del pensamiento computacional y programación de ordenadores (BASOGAIN; OLABE; OLABE, 2015). El propósito desde sus inicios, ha sido fortalecer en los estudiantes, habilidades para desarrollar programas utilizando su creatividad y apoyados en asignaturas como: las matemáticas, las ciencias y el diseño (RICO; BASOGAIN, 2018). Asimismo, se considera que los estudiantes están en capacidad de crear contenidos multimedia y; a la vez, desarrollar su competencia digital, en un mundo que exige que cada estudiantes pueda ser capaz de usar y desarrollar sus ideas a través de las diversas tecnologías (VILANOVA, 2018).

Además, es importante destacar que la gran experiencia de Inglaterra de incluir el pensamiento computacional como parte de la estructura formal del currículo, ha sido también considerada por Finlandia y Francia, quienes en estos dos últimos años han incorporado a sus planes de estudio, el pensamiento algorítmico y la programación desde los primeros grados escolares (RICO; BASOGAIN, 2018).

En el caso de América Latina, hay diversas iniciativas que están haciendo eco sobre la necesidad de incorporar el pensamiento computacional a la formación de los estudiantes en la educación básica y educación superior.

En los países de la región, la preocupación por el tema nace a la luz del protagonismo de las TIC, hacia mediados de los años noventa, cuando el fenómeno de la globalización generó que los gobiernos tomarán mayor interés por la presencia de un nuevo escenario. Por ello, los países comenzaron a diseñar políticas públicas acorde con la llamada "Sociedad de la información". Sumado a ello, la inclusión de las TIC dentro de los Objetivos de Desarrollo del Milenio de las Naciones Unidas (ODM), impulsó el protagonismo de las TIC en los diversos países (PEÑAHERRERA, 2012). Lamentablemente, este avance ha sido gradual y con dinámicas diferentes en los diferentes países, donde algunos están iniciando la fase de brindar soporte tecnológico a los sistemas educativos y otros países están iniciando la experiencia de promover el pensamiento computacional como competencia de aprendizaje de sus estudiantes.

Entre estas experiencias, Rico y Bosagain (2018) mencionan la iniciativa destacable a nivel latinoamericano desarrollada en Colombia, a través del proyecto "Introducción del pensamiento computacional en las escuelas de Bogotá y Colombia" 
(RENATA/EHU), donde se ha implementado a diversos colegios, utilizando una metodología blended o mixta basada en un ambiente virtual en la plataforma Moodle, y la participación del profesor presencial en el aula. La intención a futuro es poder integrarlo como un curso más al plan de estudios.

Otra iniciativa que se está desarrollando en Colombia, es el proyecto Coderise cuya intención es posibilitar, además de la adquisición de las habilidades del pensamiento computacional, la posibilidad de optimizar las condiciones económicas de los jóvenes a través de la promoción de emprendimientos relacionado al tema (BALLADARES; AVILÉS; PÉREZ, 2016). La iniciativa promueve

[...] el uso de Scratch en los Tecno-parques, como parte de la formación en programación y creatividad de los jóvenes. La Fundación Compartir en los Computer Clubhouse de Bogotá y la Fundación Telefónica, mediante el portal Educared, divulga y facilita talleres de formación virtual sobre Scratch (PÉREZ; ROIG-VILA, 2015, p. 7).

Este lenguaje posibilita elaborar algoritmos para crear historias interactivas, juegos y animaciones, los cuales facilitan la interacción con un conjunto de conceptos computacionales comunes a muchos lenguajes de programación (BALLADARES; AVILÉS; PÉREZ, 2016).

De acuerdo con Peñaherrera (2012) Ecuador es otro país donde se planteó la necesidad de vincular la educación con las tecnologías. La primera iniciativa oficial comenzó en el 2002, con la entrega de una PC a los maestros y la implementación de un programa de capacitación sobre el uso del ordenador. En el 2006, formalmente se afianzó la gestión de tecnologías a través de la constitución de un marco de políticas TIC. Sin embargo, pese a estos esfuerzos por dotar de infraestructura tecnológica a las escuelas y fortalecer las habilidades informacionales de los docentes, son escasos los proyectos desarrollados basados en herramientas tecnológicas del tipo Scratch. Únicamente se ha llevado a cabo un proyecto implementado por la Escuela Politécnica del Litoral (ESPOL) a nivel de educación básica y otro por la Universidad Yachay con niños de 8 a 12 años (PÉREZ; ROIG-VILA, 2015).

En Argentina, desde hace varios años, se promueven diversas políticas que estimulan el estudio de carreras de Ingeniería e Informática. Para ello, se han diseñado programas de becas que permiten estimular a los jóvenes a seguir estas profesiones. Sin embargo, el Estado argentino ha visto por necesario comenzar a trabajar con los jóvenes desde una edad más temprana en su formación. Por ello, el currículo de las escuelas secundarias está en constante cambio y las TIC se han incorporado en la escuela. Producto de ello, ha surgido el Programa Nacional "Conectar Igualdad" y los Programas de Mejoras de la escuela secundaria técnica, 
los cuales han permitido equipar a las entidades educativas con laboratorios de computadoras e infraestructura de conectividad; además, de haber permitido contar con el modelo uno a uno, que brinda una computadora por alumno (DÍAZ et al., 2014).

Con todo lo mencionado anteriormente, es evidente que hay una preocupación por el tema de las TIC, pero todavía hay una escasa atención al desarrollo del pensamiento computacional.

\section{Consideraciones finales}

La integración del pensamiento computacional a los sistemas educativos se constituye en el gran desafío para el siglo XXI. Por ello, es evidente que deben sumarse esfuerzos en la sociedad para incluirlo en los currículos educativos nacionales como una competencia indispensable en la formación de los estudiantes, la cual no debe terminar en la educación básica; sino que, debe continuar en la formación universitaria.

El pensamiento computacional no debe limitarse a la implementación de una determinada asignatura dentro del currículo, debe visualizarse como un eje transversal en el currículo que contribuya a la construcción de una ciudadanía en equidad que elimine las brechas digitales y permita la resolución de problemas a partir de la comprensión y el razonamiento.

Este nuevo escenario, exige que los docentes conozcan las diversas herramientas y lenguajes que permitan el desarrollo del pensamiento computacional. Es un gran desafío que invita a pensar en nuevas formas de dirigir los procesos de enseñanzaaprendizaje y para lograrlo se requiere el compromiso y disposición de los docentes con la finalidad de diseñar mejores prácticas educativas.

\section{Nota}

1 Organización sin fines de lucro, que tiene como objetivo incentivar a los estudiantes de colegios a aprender sobre las Ciencias Computacionales. 


\section{Referencias}

BALLADARES, J.; AVILÉS, M.; PÉREZ, H. Del pensamiento complejo al pensamiento computacional: retos para la educación contemporánea. Sophia, Colección de Filosofía de la Educación, Cuenca, v. 21, p. 143-159, 2016.

BASOGAIN, X.; OLABE, M.; OLABE, J. Pensamiento Computacional a través de la Programación: Paradigma de Aprendizaje. Revista de Educación a Distancia, Murcia, v. 46, p. 1-33, 2015. Disponible en: <http://www.redalyc.org/articulo.oa?id=54741184006>. Acceso en: 15 ago. 2018.

CARRALERO, N. Entornos para enseñar programación en secundaria. Nuevos Enfoques. QUADERNS digitales.NET, n. 70, 2011. Disponible en: <https://goo.gl/BztMsm>. Acceso en: 20 jul. 2018.

COMPAÑ, P. et al. Enseñando a programar: un camino directo para desarrollar el pensamiento computacional. Revista de Educación a Distancia, Murcia, v. 46, p. 1-15, 2015. Disponible en: <http://www.redalyc.org/articulo.oa?id=54741184011>. Acceso en: 20 ago. 2018.

DÍAZ, J. et al. Experiencias de la Facultad de informática en la enseñanza de programación en escuelas con software libre. En: DÍAZ, J. et al. Experiencias de la Facultad de informática en la enseñanza de programación en escuelas con software libre. En: CONGRESO IBEROAMERICANO DE CIENCIA, TECNOLOGÍA, INNOVACIÓN Y EDUCACIÓN, 1. Anales... Buenos Aires, 2014. p. 1-19. Disponible en: <https://www.oei.es/historico/congreso2014/01memorias2014.php>. Acceso en: 20 ago. 2018.

ESPINO, E.; GONZÁLEZ, C. Estudio sobre diferencias de género en las competencias y las estrategias educativas para el desarrollo del pensamiento computacional. Revista de Educación a Distancia, Murcia, v. 46, p. 1-20, 2015. Disponible en: <http://www.redalyc.org/articulo. oa?id=54741184012>. Acceso en: 17 ago. 2018.

LLORENS, F. et al. La enseñanza de la informática, la programación y el pensamiento computacional en los estudios preuniversitario. Education in the Knowledge Society, Salamanca, v. 18, n. 2, p. 7-17, 2017. Disponible en: <http://www.redalyc.org/articulo.oa?id=535554766001>. Acceso en: 23 ago. 2018.

PEÑAHERRERA, M. Uso de TIC en escuelas públicas de Ecuador: Análisis, reflexiones y valoraciones. Edutec - Revista Electrónica de Tecnología Educativa, Islas Baleares, n. 40, 2012. Disponible en: <http://www.edutec.es/revista/index.php/edutec-e/article/view/364/101>. Acceso en: 21 jul. 2018.

PÉREZ, H.; ROIG-VILA, R. Entornos de programación no mediados simbólicamente para el desarrollo del pensamiento computacional. Una experiencia en la formación de profesores de Informática de la Universidad Central del Ecuador. Revista de Educación a Distancia, Murcia, v. 46, p. 1-22, 2015. Disponible en: <http://www.redalyc.org/articulo.oa?id=54741184009>. Acceso en: 22 ago. 2018.

PALENCIA, M. El pensamiento computacional para potenciar el desarrollo de habilidades relacionadas con la resolución creativa de problemas. 3C TIC: Cuadernos de Desarrollo Aplicados a las TIC, Alicante, v. 6, n. 1, p. 38-63, 2017.

RICO, M.; BASOGAIN, X. Pensamiento computacional: rompiendo brechas digitales $\mathrm{y}$ educativas. EDMETIC, Córdoba, v. 7, n. 1, p. 26-42, 2018. 
RINCÓN, A. I.; ÁVILA, W. D. Una aproximación desde la lógica de la educación al pensamiento computacional. Sophia: Colección de Filosofía de la Educación, Cuenca, v. 21, n. 1, p. 161-176, 2016.

ROJAS, A.; GARCÍA, F. J. Learning Scenarios for the Subject Methodology of Programming from Evaluating the Computational Thinking of New Students. Revista Iberoamericana de Tecnologías del Aprendizaje, Vigo, v. 13, n. 1, p. 30-36, 2018.

ROMERO, M.; LEPAGE, A.; LILLE, B. Computational thinking development through creative programming in higher education. International Journal of Educational Technology in Higher Education, Nueva York, v. 14, n. 1, p. 42, 2017.

SÁEZ, J.; CÓZAR, R. Pensamiento computacional y programación visual por bloques en el aula de Primaria. Educar, Barcelona, v. 53, n. 1, p. 129-146, 2017.

SEGREDO, E.; MIRANDA, G.; LEÓN, C. Hacia la educación del futuro: El pensamiento computacional como mecanismo de aprendizaje generativo. Education in the Knowledge Society, Salamanca, v. 18, n. 2, p. 33-58, 2017.

VALVERDE, J.; FERNÁNDEZ, M.; GARRIDO, M. El pensamiento computacional y las nuevas ecologías del aprendizaje. Revista de Educación a Distancia, Murcia, v. 46, p. 1-18, 2015. Disponible en: <http://uaemex.redalyc.org/articulo.oa?id=54741184003>. Acceso en: 23 ago. 2018.

VILANOVA, G. E. Tecnología educativa para el desarrollo del pensamiento computacional. Revista Iberoamericana de Sistemas, Cibernética e Informática, Florida, v. 15, n. 3, p. 25-32, 2018. Disponible en: <http://www.iiisci.org/journal/risci/FullText.asp?var=\&id=CA074QW17>. Acceso en: 12 ago. 2018.

WING, J. M. Computational thinking and thinking about computing. Philosophical Transactions of the Royal Society of London A: Mathematical, Physical and Engineering Sciences, London, v. 366, n. 1881, p. 3717-3725, 2008.

ZAPATA, M. Pensamiento computacional: una nueva alfabetización digital. Revista de Educación a Distancia, Murcia, v. 46, p. 1-47, 2015.

ZAPOTECATL, J. L. Pensamiento Computacional. Instituto Nacional de Astrofísica, Óptica y Electrónica, Puebla, p. 1-27, 2014. Disponible en: <http://www.pensamientocomputacional.org/ Files/pensamientocomputacional.pdf>. Acceso en: 10 jul. 2018. 